\title{
BORSUK-DUGUNDJI TYPE EXTENSION THEOREMS WITH BUSEMANN CONVEX TARGET SPACES
}

\author{
Rafa Espínola, Óscar Madiedo and Adriana Nicolae \\ University of Seville, Department of Mathematical Analysis - IMUS \\ Sevilla, Spain; espinola@us.es \\ Rey Juan Carlos University, Department of Applied Mathematics, ESCET \\ 28933, Móstoles, Spain; oscar.madiedo@urjc.es \\ University of Seville, Department of Mathematical Analysis - IMUS, Sevilla, Spain \\ and Babeş-Bolyai University, Department of Mathematics \\ Kogălniceanu 1, 400084 Cluj-Napoca, Romania; anicolae@math.ubbcluj.ro
}

\begin{abstract}
In this work we study continuity properties of convex combinations in Busemann convex geodesic spaces and apply them to obtain two extension results for continuous and Lipschitz mappings with values in a Busemann convex space.
\end{abstract}

\section{Introduction}

Convex combinations in geodesic spaces are difficult elements to deal with. In a uniquely geodesic space $X$, the convex combination of two points $x_{1}, x_{2} \in X$ with coefficients $a_{1}, a_{2} \in[0,1]$ satisfying $a_{1}+a_{2}=1$ is the only point, denoted by $a_{1} x_{1} \oplus a_{2} x_{2}$, that belongs to the geodesic segment joining $x_{1}$ and $x_{2}$ with the property that $d\left(a_{1} x_{1} \oplus a_{2} x_{2}, x_{1}\right)=a_{2} d\left(x_{1}, x_{2}\right)$ and $d\left(a_{1} x_{1} \oplus a_{2} x_{2}, x_{2}\right)=a_{1} d\left(x_{1}, x_{2}\right)$. However, if we consider three points $x_{1}, x_{2}, x_{3} \in X$ and three coefficients $a_{1}, a_{2}, a_{3} \in[0,1]$ that sum to 1 , then their corresponding convex combination is not clearly defined in the same way as before since it may depend on the order of combining the points. In this case, the points

$a_{1} x_{1} \oplus\left(1-a_{1}\right)\left(\frac{a_{2}}{1-a_{1}} x_{2} \oplus \frac{a_{3}}{1-a_{1}} x_{3}\right)$ and $a_{2} x_{2} \oplus\left(1-a_{2}\right)\left(\frac{a_{1}}{1-a_{2}} x_{1} \oplus \frac{a_{3}}{1-a_{2}} x_{3}\right)$,

which may not coincide, can be, for instance, two different options for the convex combination.

The problem of finding a suitable definition for the convex combination of a finite set of points was recently taken up by Alghamdi, Kirk and Shahzad [1] in the context of Busemann convex spaces. Similar techniques were considered by Navas [18] to define a notion of barycenter and finally obtain an ergodic theorem for mappings with values in Busemann convex spaces. Both methods used in [1] and [18] actually recover ideas from [11].

Here, we follow [1] and further study, in the setting of Busemann convex spaces, convex combinations and continuity properties thereof, which allows us to obtain two Borsuk-Dugundji type extension theorems. In Section 2 we give basic definitions and properties, and address some details regarding the construction of convex combinations proposed in [1]. Section 3 mainly deals with a continuity property of

https://doi.org/10.5186/aasfm.2018.4313

2010 Mathematics Subject Classification: Primary 53C22, 54C20.

Key words: Convex combination, geodesic space, Busemann convexity, extension problem, Lipschitz mapping. 
convex combinations and briefly relates it to a corresponding one that can be obtained for an alternative notion of convex combinations defined using the approach from [18]. In Section 4 we prove counterparts of the Borsuk-Dugundji theorem for continuous and Lipschitz mappings defined on a closed subset of a metric space and taking values in a complete Busemann convex space. For the Lipschitz extension result, either the domain of the mapping or its complement are assumed to be with bounded Nagata dimension. This result is in fact a consequence of two general extension results proved by Lang and Schlichenmaier in [16] for Lipschitz mappings whose target space satisfies a Lipschitz connectedness condition that holds, in particular, in any Busemann convex space. However, our approach to the proof is to directly define the desired extension via convex combinations in the Busemann convex target space, which results in a rather simple argument and shows as well that the extension acts in a simultaneously nonexpansive way and its image belongs to the closed convex hull of the image of the original mapping.

A characterization of Nagata dimension in terms of the Lipschitz extension property is given in [7]. Spaces of finite Nagata dimension include important classes of metric spaces such as doubling ones (see [16]). Doubling metric spaces and doubling measures constitute the appropriate framework for the development of analysis in nonsmooth spaces. An excellent introduction to the needed tools and main topics in analysis in metric spaces can be found in the lecture notes by Heinonen [12] or Ambrosio and Tilli [2].

\section{Preliminaries}

Let $(X, d)$ be a metric space. For $x \in X$ and $r>0$, we denote the open ball centered at $x$ with radius $r$ by $B(x, r)$. If $A$ is a nonempty subset of $X$, the diameter of $A$ is $\operatorname{diam} A=\sup \left\{d\left(a, a^{\prime}\right): a, a^{\prime} \in A\right\}$ and the distance of a point $x \in X$ to $A$ is $d(x, A)=\inf \{d(x, a): a \in A\}$. The distance between two nonempty subsets $A$ and $B$ of $X$ is given by $d(A, B)=\inf \{d(a, b): a \in A, b \in B\}$.

Let $x, y \in X$. A geodesic path from $x$ to $y$ is a mapping $c:[0, l] \subseteq \mathbf{R} \rightarrow X$ such that $c(0)=x, c(l)=y$ and $d\left(c(t), c\left(t^{\prime}\right)\right)=\left|t-t^{\prime}\right|$ for every $t, t^{\prime} \in[0, l]$. The image of $c$ forms a geodesic segment which joins $x$ and $y$ and we denote it by $[x, y]$ when it is unique. $(X, d)$ is a (uniquely) geodesic space if every two points in $X$ can be joined by a (unique) geodesic path. A point $z \in X$ belongs to a geodesic segment joining $x$ and $y$ if and only if there exists $t \in[0,1]$ such that $d(z, x)=t d(x, y)$ and $d(z, y)=(1-t) d(x, y)$, and we write $z=(1-t) x \oplus t y$. In this case $z=c(t l)$, where $c:[0, l] \rightarrow X$ is a geodesic path from $x$ to $y$ whose image is the geodesic segment in question. A subset $A$ of $X$ is convex if it contains any geodesic segment joining each two points in $A$. More on geodesic metric spaces can be found, for instance, in $[5,6]$.

Let $(X, d)$ be a geodesic space. The metric $d: X \times X \rightarrow \mathbf{R}$ is called convex if for every $x \in X$ and every geodesic path $c:[0, l] \rightarrow X$ we have

$$
d(x, c(t l)) \leq(1-t) d(x, c(0))+t d(x, c(l)) \text { for any } t \in[0,1] .
$$

$X$ is said to be Busemann convex if given any pair of geodesic paths $c_{1}:\left[0, l_{1}\right] \rightarrow X$ and $c_{2}:\left[0, l_{2}\right] \rightarrow X$ with $c_{1}(0)=c_{2}(0)$ one has

$$
d\left(c_{1}\left(t l_{1}\right), c_{2}\left(t l_{2}\right)\right) \leq t d\left(c_{1}\left(l_{1}\right), c_{2}\left(l_{2}\right)\right) \quad \text { for any } t \in[0,1] .
$$

It is easy to see that in the definition of Busemann convexity one can drop the condition $c_{1}(0)=c_{2}(0)$. Then,

$$
d\left(c_{1}\left(t l_{1}\right), c_{2}\left(t l_{2}\right)\right) \leq(1-t) d\left(c_{1}(0), c_{2}(0)\right)+t d\left(c_{1}\left(l_{1}\right), c_{2}\left(l_{2}\right)\right) \text { for any } t \in[0,1] .
$$


A Busemann convex space is uniquely geodesic and has a convex metric. Any CAT(0) space (also known as a geodesic space of nonpositive curvature in the sense of Alexandrov) is Busemann convex. We refer to $[5,6]$ for a detailed discussion on Alexandrov spaces.

2.1. Construction of convex combinations. Let $(X, d)$ be a complete Busemann convex space and $k \geq 2$. Recall that the standard $(k-1)$-simplex, denoted by $\Delta^{k-1}$, is the set of all $k$-tuples of nonnegative numbers that sum to 1 . We describe next the construction of the convex combination introduced in [1] for a $k$-tuple $\left(x_{1}, \ldots, x_{k}\right)$ of points in $X$ and a $k$-tuple $\left(a_{1}, \ldots, a_{k}\right)$ of coefficients in $\Delta^{k-1}$. We will denote this convex combination by $\dot{\oplus}_{i=1}^{k} a_{i} x_{i}$ or simply by $\dot{\oplus} a_{i} x_{i}$ if no confusion arises. We point out some details concerning this construction that are relevant for results in this work and which were not explicitly proved in [1].

First, note that if $a_{j}=1$ for some $j$, we set $\dot{\oplus} a_{i} x_{i}=x_{j}$. Thus, we can assume in the following that there are at least two positive coefficients. We also use the notation $x_{i}^{0}$ for $x_{i}$.

For $k=2$, one has the standard definition $\dot{\oplus}_{i=1}^{2} a_{i} x_{i}=a_{1} x_{1} \oplus a_{2} x_{2}$. Now let $k=3$ and consider, for $1 \leq j \leq 3$, the sequences $\left(x_{j}^{n}\right)$ given by

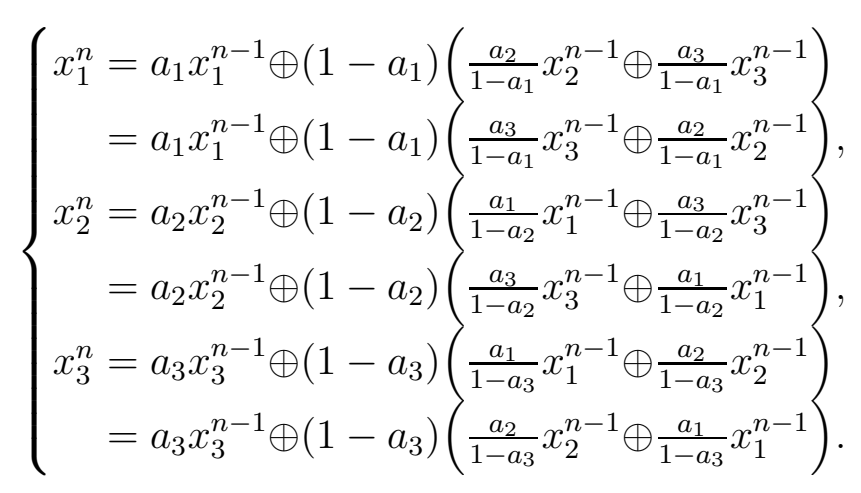

It is shown in [1] that the sequences $\left(x_{j}^{n}\right)$ converge to a same point which defines the convex combination $\dot{\oplus}_{i=1}^{3} a_{i} x_{i}$. Clearly, by the construction method, this limit is independent of the order the points $x_{i}$ are arranged in the 3-tuple (assuming the correspondence between points and coefficients is maintained). Moreover, if, for instance, $a_{3}=0$, then $\dot{\oplus}_{i=1}^{3} a_{i} x_{i}=\dot{\oplus}_{i=1}^{2} a_{i} x_{i}$. Indeed, it is immediate that $x_{1}^{1}=x_{2}^{1}=$ $x_{3}^{1}=a_{1} x_{1} \oplus a_{2} x_{2}=\dot{\oplus}_{i=1}^{2} a_{i} x_{i}$ and so $x_{j}^{n}=\dot{\oplus}_{i=1}^{2} a_{i} x_{i}$ for $1 \leq j \leq 3$ and $n \geq 1$, from where $\dot{\oplus}_{i=1}^{3} a_{i} x_{i}=\dot{\oplus}_{i=1}^{2} a_{i} x_{i}$. Additionally,

$$
d\left(\dot{\oplus}_{i=1}^{3} a_{i} x_{i}, \dot{\oplus}_{i=1}^{3} a_{i} y_{i}\right) \leq \sum_{i=1}^{3} a_{i} d\left(x_{i}, y_{i}\right) \quad \text { for any } 3 \text {-tuple }\left(y_{1}, y_{2}, y_{3}\right) \text { of points in } X \text {. }
$$

To see this, let $\left(y_{1}, y_{2}, y_{3}\right)$ be a 3 -tuple of points in $X$. Then it is an easy consequence of Busemann convexity that

$$
d\left(x_{j}^{n}, y_{j}^{n}\right) \leq a_{1} d\left(x_{1}^{n-1}, y_{1}^{n-1}\right)+a_{2} d\left(x_{2}^{n-1}, y_{2}^{n-1}\right)+a_{3} d\left(x_{3}^{n-1}, y_{3}^{n-1}\right)
$$

for $1 \leq j \leq 3$ and $n \geq 1$. Iterating, we have

$$
d\left(x_{1}^{n}, y_{1}^{n}\right) \leq a_{1} d\left(x_{1}^{n-1}, y_{1}^{n-1}\right)+a_{2} d\left(x_{2}^{n-1}, y_{2}^{n-1}\right)+a_{3} d\left(x_{3}^{n-1}, y_{3}^{n-1}\right)
$$




$$
\begin{aligned}
\leq & a_{1}\left(\sum_{i=1}^{3} a_{i} d\left(x_{i}^{n-2}, y_{i}^{n-2}\right)\right)+a_{2}\left(\sum_{i=1}^{3} a_{i} d\left(x_{i}^{n-2}, y_{i}^{n-2}\right)\right) \\
& +a_{3}\left(\sum_{i=1}^{3} a_{i} d\left(x_{i}^{n-2}, y_{i}^{n-2}\right)\right) .
\end{aligned}
$$

Using the fact that $\sum_{i=1}^{3} a_{i}=1$, we get

$$
d\left(x_{1}^{n}, y_{1}^{n}\right) \leq a_{1} d\left(x_{1}^{n-2}, y_{1}^{n-2}\right)+a_{2} d\left(x_{2}^{n-2}, y_{2}^{n-2}\right)+a_{3} d\left(x_{3}^{n-2}, y_{3}^{n-2}\right) .
$$

Continuing in this way, we finally obtain $d\left(x_{1}^{n}, y_{1}^{n}\right) \leq a_{1} d\left(x_{1}, y_{1}\right)+a_{2} d\left(x_{2}, y_{2}\right)+$ $a_{3} d\left(x_{3}, y_{3}\right)$ and we only need to take limit on $n$.

Inductively, we consider next the general case. Let $k \geq 4,\left(a_{1}, \ldots, a_{k}\right)$ be a $k$-tuple of coefficients in $\Delta^{k-1}$ such that at least two of them are positive and let $\left(x_{1}, \ldots, x_{k}\right)$ be a $k$-tuple of points in $X$. Suppose that for tuples of at most $k-1$ points in $X$, the convex combination with nonnegative coefficients that sum to 1 is defined and that it does not vary with respect to the order of points in the tuple. Assume also that the addition of a point with zero coefficient to any tuple of at most $k-2$ points leaves its convex combination unchanged. Moreover, suppose that for any $(k-1)$-tuples $\left(u_{1}, \ldots, u_{k-1}\right)$ and $\left(v_{1}, \ldots, v_{k-1}\right)$ of points in $X$ and $\left(b_{1}, \ldots, b_{k-1}\right) \in \Delta^{k-2}$,

$$
d\left(\dot{\oplus}_{i=1}^{k-1} b_{i} u_{i}, \dot{\oplus}_{i=1}^{k-1} b_{i} v_{i}\right) \leq \sum_{i=1}^{k-1} b_{i} d\left(u_{i}, v_{i}\right) .
$$

In particular, if for some $u \in X, u_{i}=u$ for all $1 \leq i \leq k-1$, then

$$
d\left(u, \dot{\oplus}_{i=1}^{k-1} b_{i} v_{i}\right) \leq \sum_{i=1}^{k-1} b_{i} d\left(u, v_{i}\right) .
$$

Take now, for $1 \leq j \leq k$, the sequences $\left(x_{j}^{n}\right)$ defined by

$$
\left\{\begin{aligned}
x_{1}^{n} & =a_{1} x_{1}^{n-1} \oplus\left(1-a_{1}\right)\left(\dot{\oplus}_{i=2}^{k} \frac{a_{i}}{1-a_{1}} x_{i}^{n-1}\right), \\
x_{2}^{n} & =a_{2} x_{2}^{n-1} \oplus\left(1-a_{2}\right)\left(\dot{\oplus}_{\left.\substack{i \neq 2 \\
i \neq 2} \frac{a_{i}}{1-a_{2}} x_{i}^{n-1}\right),}\right. \\
& \vdots \\
x_{k}^{n} & =a_{k} x_{k}^{n-1} \oplus\left(1-a_{k}\right)\left(\dot{\oplus}_{i=1}^{k-1} \frac{a_{i}}{1-a_{k}} x_{i}^{n-1}\right) .
\end{aligned}\right.
$$

Following the method given in [1] (for this one needs to apply $(2)$ ), the sequences $\left(x_{j}^{n}\right)$ converge to a same point which defines the convex combination $\dot{\oplus}_{i=1}^{k} a_{i} x_{i}$ regardless of the order the points are originally arranged. Again, if, for example, $a_{k}=0$, then

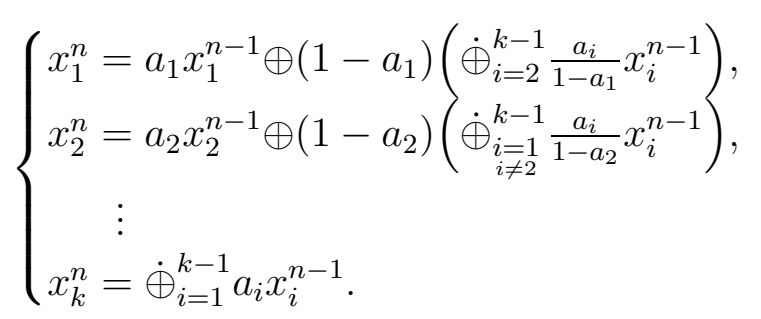

Note that the sequences $\left(x_{j}^{n}\right)$, where $1 \leq j \leq k-1$, coincide with the corresponding sequences used in the construction of $\dot{\oplus}_{i=1}^{k-1} a_{i} x_{i}$, which means that $\dot{\oplus}_{i=1}^{k} a_{i} x_{i}=\dot{\oplus}_{i=1}^{k-1} a_{i} x_{i}$. 
Furthermore,

$$
d\left(\dot{\oplus}_{i=1}^{k} a_{i} x_{i}, \dot{\oplus}_{i=1}^{k} a_{i} y_{i}\right) \leq \sum_{i=1}^{k} a_{i} d\left(x_{i}, y_{i}\right)
$$

for any $k$-tuple $\left(y_{1}, \ldots, y_{k}\right)$ of points in $X$. The above inequality follows similarly as before by applying Busemann convexity and (1). In particular, we have that

$$
d\left(x, \dot{\oplus}_{i=1}^{k} a_{i} x_{i}\right) \leq \sum_{i=1}^{k} a_{i} d\left(x, x_{i}\right) \quad \text { for any } x \in X .
$$

Note that by the argument in $[1$, p. 52], for any $n \geq 1$,

$$
\max _{1 \leq i, j \leq k} d\left(x_{i}^{n}, x_{j}^{n}\right) \leq\left(1-\sum_{i=1}^{k} a_{i}^{2}\right) \max _{1 \leq i, j \leq k} d\left(x_{i}^{n-1}, x_{j}^{n-1}\right) .
$$

2.2. Partitions of unity and spaces of finite Nagata dimension. Let $X$ be a topological space. Recall that a partition of unity on $X$ is a family $\left\{p_{i}\right\}_{i \in I}$ of continuous functions $p_{i}: X \rightarrow[0,1]$ satisfying

(i) for every $x \in X$ there exists a neighborhood $U$ of $x$ with $\left.p_{i}\right|_{U}=0$ for all but finitely many $i \in I$

(ii) $\sum_{i \in I} p_{i}=1$ and $\sup _{X} p_{i} \neq 0$ for all $i \in I$.

A partition of unity $\left\{p_{i}\right\}_{i \in I}$ on $X$ is subordinate to an open cover $\left\{U_{\alpha}\right\}_{\alpha \in \mathcal{A}}$ of $X$ if for every $i \in I$ there exists $\alpha \in \mathcal{A}$ such that $\operatorname{supp} p_{i}=\overline{\left\{x \in X: p_{i}(x) \neq 0\right\}} \subseteq U_{\alpha}$. If $X$ is in particular a metric space, then every open cover of $X$ has a subordinate partition of unity.

Let $X$ be a metric space. The Nagata dimension of $X$ introduced in [17, 3] is the least non-negative integer $n$ for which there exists a constant $c>0$ such that for all $s>0, X$ has a cover $\mathcal{B}=\left\{B_{i}\right\}_{i \in I}$ with the property that diam $B_{i} \leq c s$ for every $i \in I$ and every subset of $X$ of diameter at most $s$ meets at most $n+1$ members of $\mathcal{B}$.

Let $X$ be a metric space, $n \geq 0, A \subsetneq X$ nonempty and closed and denote $\Omega=X \backslash A$. Suppose that either $\Omega$ or $A$ have Nagata dimension $\leq n$ with a constant $c$. Then, as shown in the proofs of [16, Theorems $1.5,1.6,5.2]$, one can find two numbers $\alpha>0$ and $\delta \in(0,1)$ that only depend on $n$ and $c$ and a cover $\left\{B_{i}\right\}_{i \in I}$ of $\Omega$ by subsets of $\Omega$ such that

(i') $\operatorname{diam} B_{i} \leq \alpha d\left(B_{i}, A\right)$ for all $i \in I$;

(ii') the family $\left\{\sigma_{i}\right\}_{i \in I}$ of nonexpansive (i.e. 1-Lipschitz) functions $\sigma_{i}: \Omega \rightarrow[0, \infty$ ) defined by

$$
\sigma_{i}(x)=\max \left\{0, \delta d\left(A, B_{i}\right)-d\left(x, B_{i}\right)\right\}
$$

satisfies the property that for every $x \in \Omega, \sigma_{i}(x)>0$ for at most $n+2$ indices $i \in I$.

In this case, denoting $\bar{\sigma}=\sum_{i \in I} \sigma_{i}$, one considers the family $\left\{\varphi_{i}\right\}_{i \in I}$ of functions $\varphi_{i}: \Omega \rightarrow[0,1]$ given by

$$
\varphi_{i}(x)=\frac{\sigma_{i}(x)}{\bar{\sigma}(x)} .
$$


Then for every $x, y \in \Omega$ and all $i \in I$,

$$
\begin{aligned}
\left|\varphi_{i}(x)-\varphi_{i}(y)\right| & =\left|\frac{\sigma_{i}(x)}{\bar{\sigma}(x)}-\frac{\sigma_{i}(y)}{\bar{\sigma}(y)}\right| \leq\left|\frac{\sigma_{i}(x)}{\bar{\sigma}(x)}-\frac{\sigma_{i}(y)}{\bar{\sigma}(x)}\right|+\left|\frac{\sigma_{i}(y)}{\bar{\sigma}(x)}-\frac{\sigma_{i}(y)}{\bar{\sigma}(y)}\right| \\
& \leq \frac{1}{\bar{\sigma}(x)}\left(\left|\sigma_{i}(x)-\sigma_{i}(y)\right|+|\bar{\sigma}(x)-\bar{\sigma}(y)|\right) .
\end{aligned}
$$

Since there are at most $2 n+4$ indices $j \in I$ with $\sigma_{j}(x)>0$ or $\sigma_{j}(y)>0$, it follows that

$$
\left|\varphi_{i}(x)-\varphi_{i}(y)\right| \leq \frac{2 n+5}{\bar{\sigma}(x)} d(x, y)
$$

\section{Continuity properties of convex combinations}

We focus here on some continuity properties of convex combinations which are crucial in proving the extension theorems given in Section 4 . In the sequel we assume that $(X, d)$ is a complete Busemann convex space and $k \geq 2$.

Proposition 3.1. Let $\left(x_{1}, \ldots, x_{k}\right)$ be a $k$-tuple of points in $X$ and suppose that $\left(a_{1}, \ldots, a_{k}\right)$ and $\left(b_{1}, \ldots, b_{k}\right)$ are $k$-tuples of coefficients in $\Delta^{k-1}$. Then

$$
d\left(\dot{\oplus}_{i=1}^{k} a_{i} x_{i}, \dot{\oplus}_{i=1}^{k} b_{i} x_{i}\right) \leq\left(\prod_{i=3}^{k} i^{2}\right) \frac{D}{2} \sum_{i=1}^{k}\left|a_{i}-b_{i}\right|,
$$

where

$$
D=\max _{1 \leq i, j \leq k} d\left(x_{i}, x_{j}\right) .
$$

Proof. If $a_{j}=1$ for some $j \in\{1, \ldots, k\}$, then

$$
\begin{aligned}
d\left(\dot{\oplus}_{i=1}^{k} a_{i} x_{i}, \dot{\oplus}_{i=1}^{k} b_{i} x_{i}\right) & =d\left(x_{j}, \dot{\oplus}_{i=1}^{k} b_{i} x_{i}\right) \leq \sum_{i=1}^{k} b_{i} d\left(x_{i}, x_{j}\right)=\sum_{\substack{i=1 \\
i \neq j}}^{k} b_{i} d\left(x_{i}, x_{j}\right) \\
& \leq D \sum_{\substack{i=1 \\
i \neq j}}^{k} b_{i}=\frac{D}{2} \sum_{i=1}^{k}\left|a_{i}-b_{i}\right| .
\end{aligned}
$$

In a similar way one can show that (8) holds when $b_{j}=1$ for some $j$ and so we may assume henceforth that there are at least two positive coefficients in each $k$-tuple $\left(a_{1}, \ldots, a_{k}\right)$ and $\left(b_{1}, \ldots, b_{k}\right)$. For simplicity, we denote next $\delta=1-\sum_{i=1}^{k} b_{i}^{2}$. Note that under the previous assumption, $\delta \in(0,1)$.

If $k=2$,

$$
d\left(\dot{\oplus}_{i=1}^{2} a_{i} x_{i}, \dot{\oplus}_{i=1}^{2} b_{i} x_{i}\right)=\left|a_{1}-b_{1}\right| D=\left|a_{2}-b_{2}\right| D=\frac{D}{2} \sum_{i=1}^{2}\left|a_{i}-b_{i}\right| .
$$

Let now $k=3$ and for $1 \leq j \leq 3$ denote by $\left(x_{j}^{n}\right)$ and $\left(z_{j}^{n}\right)$ the sequences involved in the construction of the convex combinations $\dot{\oplus} a_{i} x_{i}$ and $\dot{\oplus} b_{i} x_{i}$, respectively (as explained in Section 2.1). Let also for $n \geq 0$,

$u^{n}=\frac{a_{2}}{1-a_{1}} x_{2}^{n} \oplus \frac{a_{3}}{1-a_{1}} x_{3}^{n}, v^{n}=\frac{a_{2}}{1-a_{1}} z_{2}^{n} \oplus \frac{a_{3}}{1-a_{1}} z_{3}^{n}$ and $w^{n}=\frac{b_{2}}{1-b_{1}} z_{2}^{n} \oplus \frac{b_{3}}{1-b_{1}} z_{3}^{n}$.

Then, for all $n \geq 1$,

$$
x_{1}^{n}=a_{1} x_{1}^{n-1} \oplus\left(1-a_{1}\right) u^{n-1} \quad \text { and } \quad z_{1}^{n}=b_{1} z_{1}^{n-1} \oplus\left(1-b_{1}\right) w^{n-1},
$$


and

$$
d\left(x_{1}^{n}, z_{1}^{n}\right) \leq d\left(x_{1}^{n}, a_{1} z_{1}^{n-1} \oplus\left(1-a_{1}\right) v^{n-1}\right)+d\left(a_{1} z_{1}^{n-1} \oplus\left(1-a_{1}\right) v^{n-1}, z_{1}^{n}\right) .
$$

By Busemann convexity,

$$
\begin{aligned}
d\left(x_{1}^{n}, a_{1} z_{1}^{n-1} \oplus\left(1-a_{1}\right) v^{n-1}\right) & \leq a_{1} d\left(x_{1}^{n-1}, z_{1}^{n-1}\right)+\left(1-a_{1}\right) d\left(u^{n-1}, v^{n-1}\right) \\
& \leq \sum_{i=1}^{3} a_{i} d\left(x_{i}^{n-1}, z_{i}^{n-1}\right)
\end{aligned}
$$

and

$$
\begin{aligned}
d\left(a_{1} z_{1}^{n-1} \oplus\left(1-a_{1}\right) v^{n-1}, z_{1}^{n}\right) \leq & d\left(a_{1} z_{1}^{n-1} \oplus\left(1-a_{1}\right) v^{n-1}, a_{1} z_{1}^{n-1} \oplus\left(1-a_{1}\right) w^{n-1}\right) \\
& +d\left(a_{1} z_{1}^{n-1} \oplus\left(1-a_{1}\right) w^{n-1}, z_{1}^{n}\right) \\
\leq & \left(1-a_{1}\right) d\left(v^{n-1}, w^{n-1}\right)+\left|a_{1}-b_{1}\right| d\left(z_{1}^{n-1}, w^{n-1}\right) .
\end{aligned}
$$

Since

$$
\begin{aligned}
d\left(v^{n-1}, w^{n-1}\right) & =\left|\frac{a_{2}}{1-a_{1}}-\frac{b_{2}}{1-b_{1}}\right| d\left(z_{2}^{n-1}, z_{3}^{n-1}\right) \\
& \leq \frac{\left|a_{1}-b_{1}\right|+\left|a_{2}-b_{2}\right|}{1-a_{1}} \max _{1 \leq i, j \leq 3} d\left(z_{i}^{n-1}, z_{j}^{n-1}\right)
\end{aligned}
$$

as

$$
\begin{aligned}
\left|\frac{a_{2}}{1-a_{1}}-\frac{b_{2}}{1-b_{1}}\right| & =\left|\frac{a_{2}}{1-a_{1}}-\frac{b_{2}}{1-a_{1}}+\frac{b_{2}}{1-a_{1}}-\frac{b_{2}}{1-b_{1}}\right| \\
& \leq \frac{\left|a_{2}-b_{2}\right|}{1-a_{1}}+\frac{\left|a_{1}-b_{1}\right|}{1-a_{1}} \frac{b_{2}}{1-b_{1}} \\
& \leq \frac{\left|a_{1}-b_{1}\right|+\left|a_{2}-b_{2}\right|}{1-a_{1}} \quad \text { because } \frac{b_{2}}{1-b_{1}}=\frac{b_{2}}{b_{2}+b_{3}} \leq 1
\end{aligned}
$$

and, by metric convexity,

$$
d\left(z_{1}^{n-1}, w^{n-1}\right) \leq \frac{b_{2}}{1-b_{1}} d\left(z_{1}^{n-1}, z_{2}^{n-1}\right)+\frac{b_{3}}{1-b_{1}} d\left(z_{1}^{n-1}, z_{3}^{n-1}\right) \leq \max _{1 \leq i, j \leq 3} d\left(z_{i}^{n-1}, z_{j}^{n-1}\right),
$$

it follows that

$$
d\left(x_{1}^{n}, z_{1}^{n}\right) \leq \sum_{i=1}^{3} a_{i} d\left(x_{i}^{n-1}, z_{i}^{n-1}\right)+\left(2\left|a_{1}-b_{1}\right|+\left|a_{2}-b_{2}\right|\right) \max _{1 \leq i, j \leq 3} d\left(z_{i}^{n-1}, z_{j}^{n-1}\right) .
$$

Since

$$
2\left|a_{1}-b_{1}\right|+\left|a_{2}-b_{2}\right| \leq \frac{3}{2} \sum_{i=1}^{3}\left|a_{i}-b_{i}\right| \text { and } \max _{1 \leq i, j \leq 3} d\left(z_{i}^{n-1}, z_{j}^{n-1}\right) \leq \delta^{n-1} D \text { by }(5),
$$

we obtain

$$
d\left(x_{1}^{n}, z_{1}^{n}\right) \leq \sum_{i=1}^{3} a_{i} d\left(x_{i}^{n-1}, z_{i}^{n-1}\right)+3 \delta^{n-1} \frac{D}{2} \sum_{i=1}^{3}\left|a_{i}-b_{i}\right| .
$$

In fact, one can show in this way that

$$
d\left(x_{j}^{n}, z_{j}^{n}\right) \leq \sum_{i=1}^{3} a_{i} d\left(x_{i}^{n-1}, z_{i}^{n-1}\right)+3 \delta^{n-1} \frac{D}{2} \sum_{i=1}^{3}\left|a_{i}-b_{i}\right| \quad \text { for } 1 \leq j \leq 3 .
$$


Thus,

Iterating we have

$$
\sum_{i=1}^{3} a_{i} d\left(x_{i}^{n}, z_{i}^{n}\right) \leq \sum_{i=1}^{3} a_{i} d\left(x_{i}^{n-1}, z_{i}^{n-1}\right)+3 \delta^{n-1} \frac{D}{2} \sum_{i=1}^{3}\left|a_{i}-b_{i}\right| .
$$

$$
\sum_{i=1}^{3} a_{i} d\left(x_{i}^{n}, z_{i}^{n}\right) \leq 3\left(1+\delta+\ldots+\delta^{n-1}\right) \frac{D}{2} \sum_{i=1}^{3}\left|a_{i}-b_{i}\right|
$$

and so, taking limit on $n$ and recalling that, by the Cauchy-Schwarz inequality, $1-\delta=\sum_{i=1}^{3} b_{i}^{2} \geq 1 / 3$

$$
d\left(\dot{\oplus}_{i=1}^{3} a_{i} x_{i}, \dot{\oplus}_{i=1}^{3} b_{i} x_{i}\right) \leq 9 \frac{D}{2} \sum_{i=1}^{3}\left|a_{i}-b_{i}\right| .
$$

In general, assume that (8) holds for $k-1$. Denote now for $n \geq 0$,

$$
u^{n}=\dot{\oplus}_{i=2}^{k} \frac{a_{i}}{1-a_{1}} x_{i}^{n}, \quad v^{n}=\dot{\oplus}_{i=2}^{k} \frac{a_{i}}{1-a_{1}} z_{i}^{n} \quad \text { and } \quad w^{n}=\dot{\oplus}_{i=2}^{k} \frac{b_{i}}{1-b_{1}} z_{i}^{n} .
$$

Then, for any $n \geq 1$,

$$
x_{1}^{n}=a_{1} x_{1}^{n-1} \oplus\left(1-a_{1}\right) u^{n-1} \quad \text { and } \quad z_{1}^{n}=b_{1} z_{1}^{n-1} \oplus\left(1-b_{1}\right) w^{n-1},
$$

and again, using Busemann convexity and (3),

$$
d\left(x_{1}^{n}, z_{1}^{n}\right) \leq \sum_{i=1}^{k} a_{i} d\left(x_{i}^{n-1}, z_{i}^{n-1}\right)+\left(1-a_{1}\right) d\left(v^{n-1}, w^{n-1}\right)+\left|a_{1}-b_{1}\right| d\left(z_{1}^{n-1}, w^{n-1}\right) .
$$

By the induction hypothesis,

$$
\begin{aligned}
d\left(v^{n-1}, w^{n-1}\right) & \leq\left(\prod_{i=3}^{k-1} i^{2}\right) \frac{\max _{2 \leq i, j \leq k} d\left(z_{i}^{n-1}, z_{j}^{n-1}\right)}{2} \sum_{i=2}^{k}\left|\frac{a_{i}}{1-a_{1}}-\frac{b_{i}}{1-b_{1}}\right| \\
& \leq\left(\prod_{i=3}^{k-1} i^{2}\right) \delta^{n-1} \frac{D}{2} \sum_{i=2}^{k} \frac{\left|a_{1}-b_{1}\right|+\left|a_{i}-b_{i}\right|}{1-a_{1}}
\end{aligned}
$$

and, by (4),

$$
d\left(z_{1}^{n-1}, w^{n-1}\right) \leq \sum_{i=2}^{k} \frac{b_{i}}{1-b_{1}} d\left(z_{1}^{n-1}, z_{i}^{n-1}\right) \leq \max _{1 \leq i, j \leq k} d\left(z_{i}^{n-1}, z_{j}^{n-1}\right) \leq \delta^{n-1} D .
$$

Therefore,

$$
d\left(x_{1}^{n}, z_{1}^{n}\right) \leq \sum_{i=1}^{k} a_{i} d\left(x_{i}^{n-1}, z_{i}^{n-1}\right)+k\left(\prod_{i=3}^{k-1} i^{2}\right) \delta^{n-1} \frac{D}{2} \sum_{i=1}^{k}\left|a_{i}-b_{i}\right|
$$

and similarly

$$
d\left(x_{j}^{n}, z_{j}^{n}\right) \leq \sum_{i=1}^{k} a_{i} d\left(x_{i}^{n-1}, z_{i}^{n-1}\right)+k\left(\prod_{i=3}^{k-1} i^{2}\right) \delta^{n-1} \frac{D}{2} \sum_{i=1}^{k}\left|a_{i}-b_{i}\right| \quad \text { for } 1 \leq j \leq k .
$$

Hence, we find

$$
\sum_{i=1}^{k} a_{i} d\left(x_{i}^{n}, z_{i}^{n}\right) \leq \sum_{i=1}^{k} a_{i} d\left(x_{i}^{n-1}, z_{i}^{n-1}\right)+k\left(\prod_{i=3}^{k-1} i^{2}\right) \delta^{n-1} \frac{D}{2} \sum_{i=1}^{k}\left|a_{i}-b_{i}\right|
$$


and so

$$
\sum_{i=1}^{k} a_{i} d\left(x_{i}^{n}, z_{i}^{n}\right) \leq k\left(\prod_{i=3}^{k-1} i^{2}\right)\left(1+\delta+\ldots+\delta^{n-1}\right) \frac{D}{2} \sum_{i=1}^{k}\left|a_{i}-b_{i}\right| .
$$

Passing to limit as before and using the fact that $1-\delta \geq 1 / k$ concludes the induction reasoning.

Theorem 3.2. Let $\left(x_{1}, \ldots, x_{k}\right)$ be a $k$-tuple of points in $X$ and $\left(a_{1}, \ldots, a_{k}\right)$ a $k$ tuple of coefficients in $\Delta^{k-1}$. For each $n \in \mathbf{N}$, consider the $k$-tuples $\left(z_{1}(n), \ldots, z_{k}(n)\right)$ of points in $X$ and $\left(b_{1}(n), \ldots, b_{k}(n)\right)$ of coefficients in $\Delta^{k-1}$ such that

$$
\left(z_{1}(n), \ldots, z_{k}(n)\right) \rightarrow\left(x_{1}, \ldots, x_{k}\right) \quad \text { and } \quad\left(b_{1}(n), \ldots, b_{k}(n)\right) \rightarrow\left(a_{1}, \ldots, a_{k}\right),
$$

as $n$ goes to infinity. Then $\dot{\oplus}_{i=1}^{k} b_{i}(n) z_{i}(n) \rightarrow \dot{\oplus}_{i=1}^{k} a_{i} x_{i}$, as $n$ goes to infinity.

Proof. By (3) and Proposition 3.1,

$$
\begin{aligned}
d\left(\dot{\oplus}_{i=1}^{k} b_{i}(n) z_{i}(n), \dot{\oplus}_{i=1}^{k} a_{i} x_{i}\right) \leq & d\left(\dot{\oplus}_{i=1}^{k} b_{i}(n) z_{i}(n), \dot{\oplus}_{i=1}^{k} b_{i}(n) x_{i}\right) \\
& +d\left(\dot{\oplus}_{i=1}^{k} b_{i}(n) x_{i}, \dot{\oplus}_{i=1}^{k} a_{i} x_{i}\right) \\
\leq & \sum_{i=1}^{k} b_{i}(n) d\left(z_{i}(n), x_{i}\right)+\left(\prod_{i=3}^{k} i^{2}\right) \frac{D}{2} \sum_{i=1}^{k}\left|a_{i}-b_{i}(n)\right|, \\
& \text { where } D \text { is as in Proposition 3.1. }
\end{aligned}
$$

Clearly, the above inequality proves the desired convergence.

Remark 3.3. Let $S \subseteq X$. The (closed) convex hull of $S$ is the smallest (closed) convex set containing $S$. We denote the convex hull and the closed convex hull of $S$ by $\operatorname{conv}(S)$ and $\overline{\operatorname{conv}}(S)$, respectively. Given $A \subseteq X$, let $G_{1}(A)$ be the union of all geodesic segments with endpoints in $A$. Setting, for $n \geq 2, G_{n}(S)=G_{1}\left(G_{n-1}(S)\right)$, then $\operatorname{conv}(S)=\bigcup_{n \in \mathbf{N}} G_{n}(S)$. It is easy to see that in a Busemann convex space, the closure of $\operatorname{conv}(S)$ is convex and hence coincides with $\overline{\operatorname{conv}}(S)$. As far as we know, even in the setting of $\operatorname{CAT}(0)$ spaces, it is still an open question whether $\overline{\operatorname{conv}}(S)$ is a compact set if $S$ is finite (see also the discussion in [19]).

Let $k \geq 2, S=\left\{x_{1}, \ldots, x_{k}\right\} \subseteq X$ and $\operatorname{co}(S)$ be the set of all convex combinations of $\left(x_{1}, \ldots, x_{k}\right)$ with coefficients in $\Delta^{k-1}$. Consider the onto mapping $\alpha: \Delta^{k-1} \rightarrow \operatorname{co}(S)$ defined by $\alpha\left(\left(a_{1}, \ldots, a_{k}\right)\right)=\dot{\oplus}_{i=1}^{k} a_{i} x_{i}$. Then, by Proposition 3.1, $\alpha$ is also continuous, so co $(S)$ is compact and connected as a continuous image of a compact and connected set. Note that by the construction of convex combinations, $\operatorname{co}(S) \subseteq \overline{\operatorname{conv}}(S)$, but it is not clear if these two sets are actually the same. Moreover, it is not immediate whether $\operatorname{co}(S)$ is contractible, nor if it has the fixed point property for continuous mappings.

Convex combinations can in fact be considered in the same way not only in complete Busemann convex spaces, but also in complete metric spaces where for any two points one can choose a constant speed geodesic joining them such that the resulting selection of constant speed geodesics (called a geodesic bicombing) satisfies the Busemann convexity condition. These spaces are not necessarily uniquely geodesic. More precisely, a geodesic bicombing on a metric space $(X, d)$ is a mapping $\sigma: X \times X \times[0,1] \rightarrow X$ such that for every $x, y \in X, \sigma_{x y}=\sigma(x, y, \cdot)$ is a geodesic of constant speed $d(x, y)$ from $x$ to $y$ (i.e. $d\left(\sigma_{x y}(t), \sigma_{x y}\left(t^{\prime}\right)\right)=\left|t-t^{\prime}\right| d(x, y)$ for all 
$t, t^{\prime} \in[0,1]$ and $\left.\sigma_{x y}(0)=x, \sigma_{x y}(1)=y\right)$. In addition, we assume that for any $x, y, x^{\prime}, y^{\prime} \in X$,

(i) $\sigma_{x y}(t)=\sigma_{y x}(1-t) \quad$ for all $t \in[0,1]$;

(ii) $d\left(\sigma_{x y}(t), \sigma_{x^{\prime} y^{\prime}}(t)\right) \leq(1-t) d\left(x, x^{\prime}\right)+t d\left(y, y^{\prime}\right)$ for all $t \in[0,1]$.

In this case, a set $S \subseteq X$ is called $\sigma$-convex if the image of $\sigma_{x y}$ is contained in $S$ for all $x, y \in S$ and one can define the (closed) $\sigma$-convex hull of $S$ as the smallest (closed) $\sigma$-convex set containing $S$. Note as well that the properties given in this section hold true in this more general setting.

Recently, the barycenter construction given by Navas [18] has also been considered in metric spaces with a geodesic bicombing in $[4,9]$. In fact, one could use this barycenter map to define an alternative notion of convex combinations to the one given before. Let $(X, d)$ be a complete Busemann convex space, $\mathcal{B}(X)$ the $\sigma$-algebra of Borel subsets of $X$ and denote by $P_{1}(X)$ the set of Radon probability measures of $(X, \mathcal{B}(X))$ that have finite first moment, that is,

$$
\int_{X} d(x, y) d \mu(x)<\infty
$$

for some (and hence for all) $y \in X$. Then one can construct a nonexpansive mapping bar* $\left(P_{1}(X), W\right) \rightarrow(X, d)$ which satisfies $\operatorname{bar}^{*}\left(\delta_{x}\right)=x$ for all $x \in X$, where $W$ is the 1 -Wasserstein distance and $\delta_{x}$ is the Dirac measure at $x$ (see [4, $9,18])$. In this way, it is possible to define the convex combination for a $k$-tuple $\left(x_{1}, \ldots, x_{k}\right)$ of points in $X$ and a $k$-tuple $\left(a_{1}, \ldots, a_{k}\right)$ of coefficients in $\Delta^{k-1}$ as $\operatorname{bar}^{*}\left(\sum_{i=1}^{k} a_{i} \delta_{x_{i}}\right)$. Applying a general version of the Kantorovich-Rubinstein duality theorem (see [14, Theorem 1]), one can easily show that for any $k$-tuple $\left(x_{1}, \ldots, x_{k}\right)$ of points in $X$ and any $k$-tuples $\left(a_{1}, \ldots, a_{k}\right)$ and $\left(b_{1}, \ldots, b_{k}\right)$ of coefficients in $\Delta^{k-1}$, $W\left(\sum_{i=1}^{k} a_{i} \delta_{x_{i}}, \sum_{i=1}^{k} b_{i} \delta_{x_{i}}\right) \leq(D / 2) \sum_{i=1}^{k}\left|a_{i}-b_{i}\right|$, where $D=\max _{1<i, j \leq k} d\left(x_{i}, x_{j}\right)$ (see also [9]). Thus, $d\left(\operatorname{bar}^{*}\left(\sum_{i=1}^{k} a_{i} \delta_{x_{i}}\right)\right.$, $\left.\operatorname{bar}^{*}\left(\sum_{i=1}^{k} b_{i} \delta_{x_{i}}\right)\right) \leq(D / 2) \sum_{i=1}^{k}\left|a_{i}-b_{i}\right|$, which is a continuity property similar to (8) with an improved bound. To see that this inequality is sharp, take $k=2, a_{1}=a_{2}=1 / 2$ and denote $m=\operatorname{bar}^{*}\left(\delta_{x_{1}} / 2+\delta_{x_{2}} / 2\right)$. For $b_{1}=1, b_{2}=0$, we get $d\left(m, x_{1}\right) \leq d\left(x_{1}, x_{2}\right) / 2$ and for $b_{1}=0, b_{2}=1, d\left(m, x_{2}\right) \leq$ $d\left(x_{1}, x_{2}\right) / 2$. Thus, $m=(1 / 2) x_{1} \oplus(1 / 2) x_{2}$ and in both cases equality is attained.

In contrast to this line, the approach that we followed only relies on elementary properties of Busemann convex spaces. Note that one cannot use the above upper bound in (8) as the following example shows. Consider a tripod of endpoints $x_{1}$, $x_{2}, x_{3}$ and center $o$ such that $d\left(o, x_{1}\right)=4$ and $d\left(o, x_{2}\right)=d\left(o, x_{3}\right)=1$. Then, for $a_{1}=a_{2}=a_{3}=1 / 3, b_{1}=1 / 6, b_{2}=1 / 3$ and $b_{3}=1 / 2, \dot{\oplus}_{i=1}^{3} a_{i} x_{i} \in\left[o, x_{1}\right]$ with $d\left(o, \dot{\oplus}_{i=1}^{3} a_{i} x_{i}\right)=8 / 9$ and $\dot{\oplus}_{i=1}^{3} b_{i} x_{i} \in\left[o, x_{3}\right]$ with $d\left(o, \dot{\oplus}_{i=1}^{3} b_{i} x_{i}\right)=1 / 108$. Thus,

$$
d\left(\dot{\oplus}_{i=1}^{3} a_{i} x_{i}, \dot{\oplus}_{i=1}^{3} b_{i} x_{i}\right)=\frac{97}{108}>\frac{5}{6}=\frac{D}{2} \sum_{i=1}^{3}\left|a_{i}-b_{i}\right| .
$$

Still, the obtained continuity properties are sufficient to prove the extension results given in the sequel.

\section{Borsuk-Dugundji type extension results}

This section contains our two extension results which are obtained by applying the continuity properties of convex combinations discussed previously. Note that, instead of complete Busemann convex spaces, we can actually consider mappings 
taking values in complete metric spaces with a geodesic bicombing, as pointed out before. This allows, in particular, to recover the results for Banach-valued mappings.

We prove first a counterpart of the Borsuk-Dugundji extension theorem for continuous mappings. The proof of this result follows standard patterns (see $[8$, Theorem 1.8, p. 15]).

Theorem 4.1. Let $A$ be a nonempty closed subset of a metric space $X, Y$ a complete Busemann convex space and $f: A \rightarrow Y$ a continuous mapping. Then there exists a continuous extension $F: X \rightarrow Y$ of $f$ to $X$ such that $F(X) \subseteq \overline{\operatorname{conv}}(f(A))$.

Proof. We may assume that the open set $\Omega=X \backslash A$ is nonempty. Cover $\Omega$ by balls $B_{m}=B\left(m, r_{m}\right)$ with $m \in \Omega$ and $r_{m}=d(m, A) / 3$. Note that $B_{m} \subseteq \Omega$ for each $m \in \Omega$. Let $\left\{p_{i}\right\}_{i \in I}$ be a partition of unity subordinate to this cover. For each $i \in I$ pick two points $z_{i} \in \operatorname{supp} p_{i}$ and $y_{i} \in A$ such that $d\left(y_{i}, z_{i}\right)<2 d\left(z_{i}, A\right)$.

For $x \in \Omega$, denote $I_{x}=\left\{i \in I: p_{i}(x) \neq 0\right\}$ and define

$$
F(x)= \begin{cases}f(x) & \text { if } x \in A, \\ \dot{\oplus}_{i \in I_{x}} p_{i}(x) f\left(y_{i}\right) & \text { if } x \in \Omega .\end{cases}
$$

Clearly, the mapping $F$ extends $f$ and is well-defined as $I_{x}$ is always finite for each $x \in \Omega$. We claim that $F$ is continuous. Obviously, it is continuous on the interior of $A$. We prove next the continuity on $\Omega$. For $x \in \Omega$ take a neighborhood $U$ of $x$ and a finite set $J \subseteq I$ with $\left.p_{i}\right|_{U}=0$ for all $i \in I \backslash J$. Now, for $u \in U$, it is immediate that $I_{u} \subseteq J$ and so $\dot{\oplus}_{i \in J} p_{i}(u) f\left(y_{i}\right)$ is the same convex combination that defines $F(u)$ except, at most, a finite collection of points with zero coefficients which do not modify its value as explained in Section 2.1. Since $x \in U$, this also means that $F(x)=\dot{\oplus}_{i \in J} p_{i}(x) f\left(y_{i}\right)$. Therefore, Theorem 3.2 proves the continuity at $x$.

It only rests to prove that $F$ is continuous on the boundary of $A, \partial A$. Let $m \in \partial A$ and $U_{m}$ be an open ball in $Y$ centered at $f(m)$. Take $\delta>0$ such that $f(A \cap B(m, \delta)) \subseteq U_{m}$. We check that for $m^{\prime} \in \Omega$,

$$
d\left(m, m^{\prime}\right)<\frac{\delta}{6} \quad \text { implies } \quad F\left(m^{\prime}\right) \in U_{m}
$$

which, of course, proves the claim. Notice that $F\left(m^{\prime}\right)$ is a convex combination of points $f\left(y_{i}\right)$ with $i \in I_{m^{\prime}}$, hence it suffices to prove that $f\left(y_{i}\right) \in U_{m}$ for each $i \in I_{m^{\prime}}$. To this end, given $i \in I_{m^{\prime}}$, choose $m_{i} \in \Omega$ so that supp $p_{i} \subseteq B_{m_{i}}$. Then $m^{\prime} \in B_{m_{i}}$ and we have

$$
d\left(m_{i}, A\right) \leq d\left(m_{i}, m\right) \leq d\left(m_{i}, m^{\prime}\right)+d\left(m^{\prime}, m\right)<\frac{1}{3} d\left(m_{i}, A\right)+\frac{\delta}{6} .
$$

These inequalities also imply that $d\left(m_{i}, A\right)<\delta / 4$ and $d\left(m_{i}, m\right)<\delta / 4$, from where

$$
d\left(z_{i}, m\right) \leq d\left(z_{i}, m_{i}\right)+d\left(m_{i}, m\right)<\frac{1}{3} d\left(m_{i}, A\right)+\frac{\delta}{4}<\frac{\delta}{3} .
$$

Finally, we have

$$
d\left(y_{i}, m\right) \leq d\left(y_{i}, z_{i}\right)+d\left(z_{i}, m\right)<2 d\left(z_{i}, A\right)+\frac{\delta}{3} \leq 2 d\left(z_{i}, m\right)+\frac{\delta}{3}<\delta .
$$

This means that $y_{i} \in A \cap B(m, \delta)$, so $f\left(y_{i}\right) \in U_{m}$. Hence the continuity of $F$ is proved.

We prove next a related Lipschitz extension result which also mainly follows from [16, Theorems 1.5, 1.6]. However, as mentioned in the introductory section, our proof method consists of constructing the extension in terms of convex combinations. A 
corresponding result for Banach-valued maps defined on subsets of doubling metric spaces can be found in [13, Theorem 4.1.21, p. 105].

Theorem 4.2. Let $n \geq 0$ and $A$ be a nonempty and closed subset of a metric space $X$ such that either $A$ or $X \backslash A$ has Nagata dimension $\leq n$ with a constant c. Suppose $Y$ is a complete Busemann convex space and $f: A \rightarrow Y$ an L-Lipschitz mapping. Then there exists a $C L$-Lipschitz extension $F: X \rightarrow Y$ of $f$ to $X$ such that $F(X) \subseteq \overline{\operatorname{conv}}(f(A))$, where $C \geq 1$ is a constant that depends only on $n$ and $c$.

Proof. We may assume that the open set $\Omega=X \backslash A$ is nonempty. As in Section 2.2, take $\alpha>0$ and $\delta \in(0,1)$ depending only on $n$ and $c$, a cover $\left\{B_{i}\right\}_{i \in I}$ of $\Omega$ and the family of functions $\left\{\sigma_{i}\right\}_{i \in I}$ satisfying (i') and (ii'). Moreover, consider the family of functions $\left\{\varphi_{i}\right\}_{i \in I}$ given by (6) and for each $i \in I$, let $y_{i} \in A$ such that $d\left(y_{i}, B_{i}\right) \leq(2-\delta) d\left(A, B_{i}\right)$. This point exists since $2-\delta>1$.

For $x \in \Omega$, denote $I_{x}=\left\{i \in I: \varphi_{i}(x) \neq 0\right\}$ and define

$$
F(x)= \begin{cases}f(x) & \text { if } x \in A, \\ \dot{\oplus}_{i \in I_{x}} \varphi_{i}(x) f\left(y_{i}\right) & \text { if } x \in \Omega .\end{cases}
$$

The mapping $F$ extends $f$ and is well-defined because, by (ii'), $I_{x}$ is always finite for each $x \in \Omega$. If $x \in \Omega$ and $i \in I_{x}$, then $\sigma_{i}(x)>0$, so $d\left(x, B_{i}\right)<\delta d\left(A, B_{i}\right)$ and

$$
\begin{aligned}
d\left(x, y_{i}\right) & \leq d\left(x, B_{i}\right)+\operatorname{diam} B_{i}+d\left(y_{i}, B_{i}\right) \\
& <\delta d\left(A, B_{i}\right)+\alpha d\left(A, B_{i}\right)+(2-\delta) d\left(A, B_{i}\right)=(\alpha+2) d\left(A, B_{i}\right) .
\end{aligned}
$$

We show next that $F$ is $C L$-Lipschitz. Note that in the sequel $C \geq 1$ stands for any constant that depends solely on $n$ and $c$.

Obviously, $F$ is $L$-Lipschitz on $A$. Now, for $b \in \Omega$ and $a \in A$, we have

$$
\begin{aligned}
d(F(a), F(b)) & =d\left(f(a), \dot{\oplus}_{i \in I_{b}} \varphi_{i}(b) f\left(y_{i}\right)\right) \leq \sum_{i \in I_{b}} \varphi_{i}(b) d\left(f(a), f\left(y_{i}\right)\right) \quad \text { by }(4) \\
& \leq \max _{i \in I_{b}} d\left(f(a), f\left(y_{i}\right)\right) \leq L \max _{i \in I_{b}} d\left(a, y_{i}\right) .
\end{aligned}
$$

If $i \in I_{b}$, then $d\left(A, B_{i}\right) \leq d\left(a, B_{i}\right) \leq d(a, b)+d\left(b, B_{i}\right)<d(a, b)+\delta d\left(A, B_{i}\right)$ and this yields $d\left(A, B_{i}\right)<(1-\delta)^{-1} d(a, b)$. Using (9), we then get

$$
d\left(a, y_{i}\right) \leq d(a, b)+d\left(b, y_{i}\right)<d(a, b)+(\alpha+2) d\left(A, B_{i}\right)<\left(1+\frac{\alpha+2}{1-\delta}\right) d(a, b) .
$$

Thus, $d(F(a), F(b)) \leq C L d(a, b)$.

Let next $a, b \in \Omega$. Suppose first that there exists $j \in I_{a} \cap I_{b}$. By (ii'), the set $I_{a} \cup I_{b}$ contains at most $2 n+3$ indices $i \in I$. Moreover, $F(a)=\dot{\oplus}_{i \in I_{a} \cup I_{b}} \varphi_{i}(a) f\left(y_{i}\right)$ and $F(b)=\dot{\oplus}_{i \in I_{a} \cup I_{b}} \varphi_{i}(b) f\left(y_{i}\right)$ since we are not adding more than a finite collection of points with zero coefficients which do not change a convex combination (see Section 2.1). By the Cauchy-Schwarz inequality, $\sum_{i \in I_{a} \cup I_{b}} \varphi_{i}(a)^{2} \geq 1 /(n+2)$. Applying Proposition 3.1 we obtain that

$$
d(F(a), F(b)) \leq C \max _{i, k \in I_{a} \cup I_{b}} d\left(f\left(y_{i}\right), f\left(y_{k}\right)\right) \sum_{i \in I_{a} \cup I_{b}}\left|\varphi_{i}(a)-\varphi_{i}(b)\right| .
$$

Note that

$$
\max _{i, k \in I_{a} \cup I_{b}} d\left(f\left(y_{i}\right), f\left(y_{k}\right)\right) \leq L \max _{i, k \in I_{a} \cup I_{b}} d\left(y_{i}, y_{k}\right) \leq 2 L \max _{i \in I_{a} \cup I_{b}} d\left(y_{i}, y_{j}\right) .
$$

Let $i \in I_{a}$. Since

$$
d\left(A, B_{j}\right) \leq d\left(y_{i}, B_{j}\right) \leq d\left(y_{i}, a\right)+d\left(a, B_{j}\right)<(\alpha+2) d\left(A, B_{i}\right)+\delta d\left(A, B_{j}\right) \quad \text { by }(9),
$$


it follows that $d\left(A, B_{j}\right) \leq(\alpha+2)(1-\delta)^{-1} d\left(A, B_{i}\right)$. Similarly,

$$
d\left(A, B_{i}\right) \leq(\alpha+2)(1-\delta)^{-1} d\left(A, B_{j}\right)
$$

Then, again by (9),

$$
\begin{aligned}
d\left(y_{i}, y_{j}\right) & \leq d\left(y_{i}, a\right)+d\left(a, y_{j}\right) \leq(\alpha+2)\left(d\left(A, B_{i}\right)+d\left(A, B_{j}\right)\right) \\
& \leq(\alpha+2)\left(1+\frac{\alpha+2}{1-\delta}\right) d\left(A, B_{j}\right) .
\end{aligned}
$$

Note that the above inequality can also be proved by the same argument in the case $i \in I_{b}$. By (7),

$$
d(F(a), F(b)) \leq C L d\left(A, B_{j}\right) \sum_{i \in I_{a} \cup I_{b}} \frac{d(a, b)}{\bar{\sigma}(a)} .
$$

Let $m \in I$ such that $a \in B_{m}$. Then $m \in I_{a}$ and

$$
\bar{\sigma}(a) \geq \sigma_{m}(a)=\delta d\left(A, B_{m}\right) \geq \delta \frac{1-\delta}{\alpha+2} d\left(A, B_{j}\right) .
$$

This shows that $d(F(a), F(b)) \leq C L d(a, b)$.

Suppose now $I_{a} \cap I_{b}=\emptyset$. Since $a \in B_{m}$ for some $m \in I_{a}$, we then have that $\sigma_{m}(b)=0$, which yields $d\left(A, B_{m}\right) \leq \delta^{-1} d\left(b, B_{m}\right) \leq \delta^{-1} d(b, a)$. Applying (9),

$$
d\left(a, y_{m}\right) \leq(\alpha+2) d\left(A, B_{m}\right) \leq \frac{\alpha+2}{\delta} d(a, b) .
$$

Hence,

$$
\begin{aligned}
d(F(a), F(b)) & \leq d\left(F(a), F\left(y_{m}\right)\right)+d\left(F\left(y_{m}\right), F(b)\right) \leq C L\left(d\left(a, y_{m}\right)+d\left(y_{m}, b\right)\right) \\
& \leq C L\left(2 d\left(a, y_{m}\right)+d(a, b)\right) \leq C L d(a, b),
\end{aligned}
$$

where the last inequality follows using (10) and adjusting the constant $C$.

Remark 4.3. The extensions constructed in Theorems 4.1 and 4.2 act in a simultaneously nonexpansive way, that is, for $f$ and $g$ continuous (resp. Lipschitz) mappings from $A$ to $Y$ we have that

$$
\sup _{x \in X} d(F(x), G(x)) \leq \sup _{x \in A} d(f(x), g(x)),
$$

which is an immediate consequence of (3).

Thus, considering the multivalued extension operator which assigns to every bounded continuous (resp. bounded $L$-Lipschitz) mapping all its bounded continuous (resp. bounded $C L$-Lipschitz) extensions, one can find a nonexpansive selection of it with respect to the supremum norm. Recent results on parameter dependence of extensions of Lipschitz mappings have been obtained in the setting of Hilbert spaces in [15] and in Alexandrov spaces in [10].

Acknowledgements. R. Espínola and A. Nicolae have been partially supported by DGES (MTM2015-65242-C2-1-P). A. Nicolae would also like to acknowledge the Juan de la Cierva - Incorporación Fellowship Program of the Spanish Ministry of Economy and Competitiveness. The research of Ó. Madiedo was supported in part by MINECO (Spain), project MTM2015-65825-P and the Red de Institutos Universitarios de Matemáticas (REDIum). Different parts of this paper were developed during a visit of Ó. Madiedo to IMUS (Sevilla). The authors would also like to thank the referees for their comments and suggestions that helped improve the bound in Proposition 3.1. 


\section{References}

[1] Alghamdi, M. A., W. A. Kirk, and N. Shahzad: Remarks on convex combinations in geodesic spaces. - J. Nonlinear Convex Anal. 15, 2014, 49-59.

[2] Ambrosio, L, and P. Tilli: Topics on analysis in metric spaces. - Oxford Lecture Ser. Math. Appl. 25, Oxford Univ. Press, Oxford, 2004.

[3] Assound, P.: Sur la distance de Nagata. - C. R. Acad. Sci. Paris Sér. I Math. 294, 1982, 31-34.

[4] BAsso, G.: Fixed point theorems for metric spaces with a conical geodesic bicombing. - Ergodic Theory Dynam. Systems (to appear).

[5] Bridson, M. R., and A. Haefliger: Metric spaces of non-positive curvature. - SpringerVerlag, Berlin, 1999.

[6] Burago, D., Y. Burago, and S. Ivanov: A course in metric geometry. - Grad. Stud. Math. 33, Amer. Math. Soc., Providence, RI, 2001.

[7] Brodskiy, N., J. Dydak, J. Higes, and A. Mitra: - Assouad-Nagata dimension via Lipschitz extensions. - Israel J. Math. 171, 2009, 405-423.

[8] BRUdnyi, A., and Y. BRUdnyi: Methods of geometric analysis in extension and trace problems. Volume 1. - Monogr. Math. 102, Birkhäuser/Springer Basel AG, Basel, 2012.

[9] Descombes, D.: Asymptotic rank of spaces with bicombings. - Math. Z. 284, 2016, 947-960.

[10] Espínola, R., and A. Nicolae: Continuous selections of Lipschitz extensions in metric spaces. - Rev. Mat. Complut. 28, 2015, 741-759.

[11] Es-Sahib, A., and H. Heinich: Barycentre canonique pour un espace métrique à courbure négative. - In: Séminaire de Probabilités XXXIII, Lecture Notes in Math. 1709, Springer, 1999, $355-370$.

[12] Heinonen, J.: Lectures on analysis on metric spaces. - Springer-Verlag, New York, 2001.

[13] Heinonen, J., P. Koskela, N. Shanmugalingam, and J. T. Tyson: Sobolev spaces on metric measure spaces: An approach based on upper gradients. - New Math. Monogr. 27, Cambridge Univ. Press, 2015.

[14] Kellerer, H. G.: Duality theorems and probability metrics. - In: Proceedings of the seventh conference on probability theory (Braşov, 1982), VNU Sci. Press, Utrecht, 1985, 211-220.

[15] KopeckÁ, E.: Bootstrapping Kirszbraun's extension theorem. - Fund. Math. 217, 2012, $13-18$.

[16] LAng, U., and T. Schlichenmaier: Nagata dimension, quasisymmetric embeddings, and Lipschitz extensions. - Int. Math. Res. Not. 2005, 2005, 3625-3655.

[17] NagAta, J.-I.: Note on dimension theory for metric spaces. - Fund. Math. 45, 1958, $143-181$.

[18] NAvAs, A.: An $L^{1}$ ergodic theorem with values in a non-positively curved space via a canonical barycenter map. - Ergodic Theory Dynam. Systems 33, 2013, 609-623.

[19] Petrunin, A.: Convex hull in CAT(0). - MathOverflow, http://mathoverflow.net/q/6627. 